\title{
Detection of enteroviruses during a 2018 hand, foot and mouth disease outbreak in Malaysia
}

\author{
Lee, M.H.P. ${ }^{1}$, Chong, Y.M. ${ }^{1}$, Tay, C.G. ${ }^{2}$, Koh, M.T. ${ }^{2}$, Chem, Y.K. ${ }^{3}$, Noordin, N. ${ }^{3}$, Jahis, R. ${ }^{4}$, Sam, I.C. ${ }^{1}$, \\ Chan, Y.F. ${ }^{1 *}$
}

${ }^{1}$ Department of Medical Microbiology, Faculty of Medicine, University of Malaya, 50603 Kuala Lumpur, Malaysia

${ }^{2}$ Department of Paediatrics, Faculty of Medicine, University of Malaya, 50603 Kuala Lumpur, Malaysia

${ }^{3}$ National Public Health Laboratory, Sungai Buloh, Selangor, Malaysia

${ }^{4}$ Zoonosis Sector, Disease Control Division, Ministry of Health, Putrajaya, Malaysia

*Corresponding author: chanyf@um.edu.my

\section{ARTICLE HISTORY}

Received: 3 August 2020

Revised: 5 December 2020

Accepted: 6 December 2020

Published: 25 March 2021

\begin{abstract}
Hand, foot, and mouth disease (HFMD) is a common childhood disease caused by enteroviruses. In 2018, a HFMD outbreak in Malaysia affected over 76,000 children. In this study, we used RT-qPCR and CODEHOP PCR to detect the causative agents in 89 clinically diagnosed HFMD patients in Kuala Lumpur and Selangor. Most (62.9\%) of the children were below 3 years old. PCR with either assay detected enteroviruses in 84.2\% (75/89) and CODEHOP PCR successfully typed $66.7 \%$ (50/75) of the enteroviruses. Sequencing of CODEHOP amplicons showed co-circulation of multiple enteroviruses with coxsackievirus A6 (CV-A6) and A16 as the predominant serotypes, but not the neurovirulent enterovirus A71. CV-A6 infection was more common in children less than 12 months old $(p=0.01)$ and was more likely to cause vesicles in the gluteal area $(p=0.01)$ compared to other enteroviruses. Establishing a robust identification method during HFMD outbreaks is important for patient management and public health responses.
\end{abstract}

Keywords: hand, foot and mouth disease; HFMD; enterovirus; coxsackievirus A6; Malaysia.

\section{INTRODUCTION}

Hand, foot and mouth disease (HFMD) is the second most common infectious diseases in Malaysia ( $\mathrm{MOH}, 2019)$. In Malaysia, enterovirus 71 (EV-A71), coxsackievirus A16 (CV-A16) and CV-A6 have been reported as causative agents of HFMD outbreaks (Chan et al., 2012; Aw-Yong et al., 2017). However, the disease is caused by a group of enteroviruses with over 100 serotypes. Since virus surveillance is limited, the enteroviruses that cause yearly HFMD epidemics remain unknown.

Within the genus of enteroviruses, EV-A71 and some echoviruses cause neurological complications aside from polioviruses (Solomon et al., 2010; Bubba et al., 2020). Quick identification of these neurotropic enteroviruses is important for patient management. CV-A16 and EV-A71 are the major causative agents of HFMD in China and have been endemic in Southeast Asia and the Pacific region for two decades (Van Tu et al., 2007; Iwai et al., 2009). However since 2011, outbreaks of other enterovirus strains such as CV-A6 and CV-A10 have been reported in China (He et al., 2017), Finland (Blomqvist et al., 2010), France (Mirand et al., 2016), Japan (Fujimoto et al., 2012), United Kingdom (Gaunt et al., 2015) and Taiwan (Wei et al., 2011). An unusual CV-A2 circulation was also recently documented in China (Yang et al., 2018). With co-circulation of many enteroviruses at any one time, the lack of viral surveillance for HFMD in Malaysia hampers the understanding of HFMD epidemiology, which in turn impacts appropriate public health and resource management, including the development of multivalent vaccines and introduction of EV-A71 vaccines.

Routine identification is usually based upon virus isolation followed by immunofluorescence. These are timeconsuming and labour-intensive, requiring 7-14 days, and hence do not provide a rapid laboratory diagnosis. Molecular diagnosis based on real-time PCR is time-saving and sensitive (Nijhuis et al., 2002; Robinson et al., 2002). The COnsensus-DEgenerate Hybrid Oligonucleotide Primer (CODEHOP) strategy was developed to detect and identify distant-related pathogens (Rose et al., 2003). When applied to enteroviruses, CODEHOP PCR uses highly degenerate primers targeting VP1 to identify different serotypes compared to traditional specific primers (Nix et al., 2006; Chiang et al., 2012). Therefore, the aim of this study is to describe the use of both real-time PCR and CODEHOP PCR to detect and identify the serotypes of enteroviruses in HFMD patients during an outbreak in Malaysia in 2018. 


\section{MATERIALS AND METHODS}

Patients presenting with HFMD at the University Malaya Medical Centre (UMMC), a teaching hospital in Kuala Lumpur from June and July 2018 were included in this study. These patients presented with clinical features consistent with HFMD which include acute febrile illness accompanied by vesicular and tender rash over, but not limited to, the palms/ soles with or without intraoral ulcers. Clinical data and patient demographics were obtained. Throat swabs were collected and transported in virus transport medium (VTM).

VTM received were filtered with a $0.45 \mu \mathrm{m}$ syringe filter. RNA was extracted from $280 \mu \mathrm{l}$ VTM using QIAamp Viral RNA mini kit (QIAGEN, Germany) according to the manufacturer's instructions. Primer and probe sequences were from Thanh et al. (2015) (Table 1). RT-qPCR reaction consisted of $4 \times$ TaqMan Fast Virus 1-Step Mastermix (Thermo Fisher Scientific, USA), $0.5 \mu \mathrm{M}$ of each primer (ENT-F and ENT-R), $0.25 \mu \mathrm{M}$ of ENT probe and $5 \mu \mathrm{l}$ of RNA template. The reaction mixes were subjected to $50^{\circ} \mathrm{C}$ for 5 minutes for cDNA synthesis, $95^{\circ} \mathrm{C}$ for $20 \mathrm{~s}$, followed by 40 cycles of $95^{\circ} \mathrm{C}$ for $3 \mathrm{~s}$ and $60^{\circ} \mathrm{C}$ for $30 \mathrm{~s}$. RT-qPCRs were performed using StepOne Plus Real-Time PCR (Applied Biosystems, USA) and analyzed using StepOne Plus Software version 2.3.

For CODEHOP PCR (Nix et al., 2006), synthesis of cDNA was carried out in a $10 \mu \mathrm{l}$ mixture containing $4.5 \mu \mathrm{l}$ of RNA, $100 \mu \mathrm{M}$ deoxynucleoside triphosphate (dNTP), 5X first-strand buffer, $0.01 \mathrm{M}$ dithiothreitol (DTT), $0.5 \mu \mathrm{M}$ cDNA primer mixture (AN32, AN33, AN34, AN35), $20 \mathrm{U}$ of RNaseOUT and $100 \mathrm{U}$ of SuperScript III reverse-transcriptase (Invitrogen, USA). Following incubation at $22^{\circ} \mathrm{C}$ for $10 \mathrm{~min}, 42^{\circ} \mathrm{C}$ for $45 \mathrm{~min}, 95^{\circ} \mathrm{C}$ for $5 \mathrm{~min}, 5 \mu \mathrm{l}$ of the RT reaction mixture was then used in the first PCR (PCR1), consisting of 2X MyTaq reaction buffer, $5 \mathrm{U}$ of MyTaq DNA polymerase (Bioline, UK), $400 \mathrm{nM}$ each of primers 222 and 224 , with 40 cycles of amplification $\left(95^{\circ} \mathrm{C}\right.$ for $15 \mathrm{~s}, 42^{\circ} \mathrm{C}$ for $15 \mathrm{~s}, 72^{\circ} \mathrm{C}$ for $\left.15 \mathrm{~s}\right)$. One microliter of the first PCR was added to a second PCR (PCR2) for semi-nested amplification. PCR2 contained primers AN88 and AN89, and the reaction mixture was the same as PCR 1 , with 40 cycles $\left(95^{\circ} \mathrm{C}\right.$ for $15 \mathrm{~s}$, $60^{\circ} \mathrm{C}$ for $15 \mathrm{~s}, 72^{\circ} \mathrm{C}$ for $15 \mathrm{~s}$ ). The amplicons were separated and visualized in $1.5 \%$ agarose gel containing GelRed and were gel purified using DNA Clean \& Concentrator (Zymo, USA) prior to Sanger sequencing (Apical Scientific Sdn. Bhd). Alignment of the sequences was performed using Geneious Prime 2020 (Biomatters Inc., New Zealand). VP1 nucleotide sequences were checked against the NCBI database by Blast search to determine the enterovirus serotype with the highest identity.

To compare the sensitivity of RT-qPCR and CODEHOP PCR, both assays were performed as described above with enterovirus control RNA from in vitro transcribed EV-A71 (Tan et al., 2016). Ten-fold serially diluted RNA copy numbers were assayed with 6 technical replicates with both RT-qPCR and CODEHOP PCR.

The associations of enterovirus PCR positivity with age groups, gender and clinical symptoms were determined by Chi-square test with SPSS version 23.0 (IBM, USA), and $p<0.05$ was regarded as statistically significant. This study was approved by the UMMC Medical Ethics Committee (reference number: 932.17). Our institution does not require informed consent for retrospective studies of anonymised samples.

\section{RESULTS AND DISCUSSION}

During 2018, a major HFMD outbreak occurred across Malaysia with over 76,000 infected (MOH, 2019). We tested 89 samples collected from UMMC during the outbreak period. The median
Table 1. Primers and probes used for molecular detection in CODEHOP and RT-qPCR

\begin{tabular}{|c|c|c|c|}
\hline Primers & Sequences $\left(5^{\prime}-3^{\prime}\right)$ & $\begin{array}{l}\text { Target } \\
\text { region }\end{array}$ & Polarity \\
\hline CODEHOP & & AN32 & GTYTGCCA \\
\hline VP1 & antisense & & \\
\hline AN33 & GAYTGCCA & VP1 & antisense \\
\hline AN34 & CCRTCRTA & VP1 & antisense \\
\hline AN35 & RCTYTGCCA & VP1 & antisense \\
\hline 222 & CICCIGGIGGIAYRWACAT & VP1 & antisense \\
\hline 224 & GCIATGYTIGGIACICAYRT & VP3 & sense \\
\hline AN88 & CCAGCACTGACAGCAGYNGARAYNGG & VP1 & antisense \\
\hline AN89 & TACTGGACCACCTGGNGGNAYRWACAT & VP1 & sense \\
\hline \multicolumn{4}{|l|}{ RT-qPCR } \\
\hline ENT-F & CCCTGAATGCGGCTAAT & 5' UTR & sense \\
\hline ENT-R & ATTGTCACCATAAGCAGCC & 5' UTR & antisense \\
\hline ENT probe & $\begin{array}{l}\text { ATTO550N-ACCCAAAGTAGTCGGTTCCG- } \\
\text { IAbRQSp }\end{array}$ & & \\
\hline
\end{tabular}

Primer and probe sequences for CODEHOP and RT-qPCR were from Nix et al. (2006) and Thanh et al. (2015), respectively.

ATTO 550N is the 5' fluorophore and IAbRQSp (lowa Black RQ) is the 3' quencher.

age of the patients was 27 months (range 4 months -10.6 years). The suspected HFMD patients comprised of 45 males $(50.6 \%)$ and 44 females (49.4\%). The RT-qPCR based on the 5 -untranslated regions detected enteroviruses in $83.1 \%$ $(74 / 89)$ of the samples. By utilising a quick and sensitive RT-qPCR, patients with suspected HFMD could be diagnosed in two hours. CODEHOP PCR amplicons which require sequencing will take at least an additional day. Early and timely detection could aid in the control of the spread of HFMD among susceptible children especially those attending preschools.

By using the CODEHOP PCR, we detected a total of $56.2 \%$ enteroviruses (50/89). Of these, $55.1 \%(49 / 89)$ were positive with both assays, and one sample was detected only with CODEHOP but not RT-qPCR. Therefore, a total of 75/89 (84.3\%) of samples were positive with either or both PCR assays. We found that the limits of detection for RT-qPCR and CODEHOP were $10^{2}$ RNA copies per reaction and $10^{3}$ RNA copies per reaction respectively, which explained the lower sensitivity of CODEHOP PCR compared to RT-qPCR. Despite its lower sensitivity, the 350-400 bp amplicon from CODEHOP PCR can be sequenced to type the enterovirus species. In total, $44 \%$ CV-A6 (22/50), 40\% CV-A16 (20/50), 10\% EV-A71 (5/50), 2\% CV-A10 $(1 / 50)$ and $4 \%$ CV-B3 $(2 / 50)$ were sequenced from the CODEHOP PCR amplicons. Nine samples positive for CODEHOP PCR remained undetermined as the sequences had similarity to human genes, which could be due to the degeneracy properties of the CODEHOP primers. A positive CODEHOP amplicon therefore should be sequenced for confirmation of enterovirus species. The National Public Health Laboratory collected 2236 samples for the same duration of this 2018 HFMD outbreak and showed $32.96 \%$ enterovirus positive (737/2238); with 30.53\% EV-A71 (225/737), 32.29\% CV-A16 (238/737) and $37.18 \%$ other enteroviruses (273/737)) (personal communication). Similar to the current study, more robust typing will be required to type the enteroviruses to determine the presence of other enteroviruses.

Univariate analysis was performed to determine the correlation between age, gender and clinical symptoms with PCR-positivity for enteroviruses (Table 2). Children $\leq 12$ months were significantly more likely to be infected by CV-A6 (47.6\%) than by CVA16 $(10.5 \%)$ or other EV (16.7\%) (Table 2). Patients 
Table 2. Comparison of demographic and clinical features of patients with CV-A6, CV-A16 and other enterovirus infections

\begin{tabular}{lccc}
\hline & CVA6 $(\mathrm{n}=22)$ & CVA16 $(\mathrm{n}=20)$ & Other EV $^{*}(\mathrm{n}=33)$ \\
\hline Age (months) & & & \\
$\leq 12$ months & $10(47.6 \%)$ & $2(10.5 \%)$ & $5(16.7 \%)$ \\
$>12$ months & $11(52.4 \%)$ & $17(89.5 \%)$ & $25(83.3 \%)$ \\
Unknown & $1(4.5 \%)$ & $1(5.0 \%)$ & $3(0.9 \%)$ \\
Sex & & & \\
Male & $9(40.9 \%)$ & $9(45.0 \%)$ & $17(51.5 \%)$ \\
Female & $13(59.1 \%)$ & $11(55.0 \%)$ & $16(48.5 \%)$ \\
Signs and symptoms & & & \\
Fever & $18(81.8 \%)$ & $10(50.0 \%)$ & $21(63.6 \%)$ \\
Mouth ulcers & $18(81.8 \%)$ & $15(75.0 \%)$ & $22(66.7 \%)$ \\
Vesicles on hand & $16(72.7 \%)$ & $14(70.0 \%)$ & $18(54.5 \%)$ \\
Vesicles on feet & $16(72.7 \%)$ & $7(80.0 \%)$ & $20(60.6 \%)$ \\
Vesicles on buttocks & $12(54.5 \%)$ & $5(15.2 \%)$ & 0.73 \\
\hline
\end{tabular}

*Other enteroviruses include EV-A71 (5), CV-A10 (1), CV-B3 (2) and non-typable enteroviruses (25). \#Chi-square test was used. Significant $p$-values $(p<0.05)$ are indicated in bold.

with CV-A6 infection showed significantly more vesicles on the gluteal area than other enterovirus infections $(p=0.01)$, which supports previous reports (Mirand et al., 2016; Uppala et al., 2018).

The number of CV-A6 detected in Malaysia has been increasing since 2012 (Aw-Yong et al., 2017). An increase in CV-A6 infections have been reported both within and outside the Asia Pacific region (Fujimoto et al., 2012; Puenpa et al., 2014). The main circulating viral causes of HFMD in Malaysia had been EV-A71 for many years since the first major outbreak in the Asia Pacific region in 1997 (Chan et al., 2000) and CVA16, but since 2012 CV-A6 has become a major contributor (Aw-Yong et al., 2017). There has been a corresponding decline of EV-A71 in Asia since the last fatal outbreak in Cambodia in 2012 (Duong et al., 2016), although it still contributes to neurological cases in Europe (Bubba et al., 2020). Therefore, it is essential to type other enteroviruses with tools such as CODEHOP PCR.

In conclusion, our findings showed that CV-A6 has emerged and co-circulated with other enteroviruses during the 2018 outbreak in Malaysia. The co-circulation of various enteroviruses highlights the dire need for a multivalent HFMD vaccine. The usage of RT-qPCR and CODEHOP PCR in this study are efficient methods for detection and surveillance of emerging enteroviruses, in order to monitor and initiate early control for the disease. The early and timely detection and identification of the circulating enterovirus will greatly assist national policy and decision makers on deciding the most suitable HFMD vaccine when is available in the near future.

\section{ACKNOWLEDGEMENTS}

This study was supported by grants from the Ministry of Higher Education, Malaysia (Fundamental Research Grant Scheme FRGS/1/2018/SKK11/UM/02/2) and Ministry of Science, Technology and Innovation (E-Science fund 02-01-03-SF1210). Authors also acknowledge the valuable contributions of Hien Fuh Ng and Thinesshwary Yogarajah. The authors declare no competing interests.

\section{Conflict of interest}

The authors declare that they have no conflict of interest.

\section{REFERENCES}

Aw-Yong, K.L., Sam, I.C., Koh, M.T. \& Chan, Y.F. (2017). Causative agents of hand, foot and mouth disease in University Malaya Medical Centre, Kuala Lumpur, Malaysia in 20122013. Tropical Biomedicine 34: 240-248.

Blomqvist, S., Klemola, P., Kaijalainen, S., Paananen, A., Simonen, M.L., Vuorinen, T. \& Roivainen, M. (2010). Cocirculation of coxsackieviruses $A 6$ and $A 10$ in hand, foot and mouth disease outbreak in Finland. Journal of Clinical Virology 48: 49-54. https://doi.org/10.1016/j.jcv.2010.02.002

Bubba, L., Broberg, E.K., Jasir, A., Simmonds, P. \& Harvala, H., on behalf of the Enterovirus study collaborators (2020). Circulation of non-polio enteroviruses in $24 \mathrm{EU}$ and EEA countries between 2015 and 2017: A retrospective surveillance study. Lancet Infectious Diseases 20: 350-361. https://doi.org/10.1016/S1473-3099(19)30566-3

Chan, L.G., Parashar, U.D., Lye, M.S., Ong, F.G., Zaki, S.R., Alexander, J.P., Ho, K.K., Han, L.L., Pallansch, M.A., Suleiman, A.B. \& Jegathesan, M. (2000). Deaths of children during an outbreak of hand, foot, and mouth disease in Sarawak, Malaysia: clinical and pathological characteristics of the disease for the outbreak study group. Clinical Infectious Diseases 31: 678-683. https://doi.org/10.1086/314032

Chan, Y.F., Wee, K.L., Chiam, C.W., Khor, C.S., Chan, S.Y., Amalina, W.M.Z. \& Sam, I.C. (2012). Comparative genetic analysis of VP4, VP1 and 3D gene regions of enterovirus 71 and coxsackievirus A16 circulating in Malaysia between 19972008. Tropical Biomedicine 29: 451-466.

Chiang, P.S., Huang, M.L., Luo, S.T., Lin, T.Y., Tsao, K.C. \& Lee, M.S. (2012). Comparing molecular methods for early detection and serotyping of enteroviruses in throat swabs of pediatric patients. PLoS One 7: e48269. https://doi.org/ 10.1371/journal.pone.0048269

Duong, V., Mey, C., Eloit, M., Zhu, H., Danet, L., Huang, Z., Zou, G., Tarantola, A., Cheval, J. \& Perot, P. (2016). Molecular epidemiology of human enterovirus 71 at the origin of an epidemic of fatal hand, foot and mouth disease cases in Cambodia. Emerging Microbes \& Infections 5: e104. https:// doi.org/10.1038/emi.2016.101

Fujimoto, T., lizuka, S., Enomoto, M., Abe, K., Yamashita, K., Hanaoka, N., Okabe, N., Yoshida, H., Yasui, Y. \& Kobayashi, M. (2012). Hand, foot, and mouth disease caused by coxsackievirus A6, Japan, 2011. Emerging Infectious Diseases 18: 337-339. https://dx.doi.org/10.3201/eid1802.111147 
Gaunt, E., Harvala, H., Osterback, R., Sreenu, V.B., Thomson, E., Waris, M. \& Simmonds, P. (2015). Genetic characterization of human coxsackievirus $A 6$ variants associated with atypical hand, foot and mouth disease: a potential role of recombination in emergence and pathogenicity. Journal of General Virology 96: 1067-1079. https://doi.org/ 10.1099/vir.0.000062

He, S.Z., Chen, M.Y., Xu, X.R., Yan, Q., Niu, J.J., Wu, W.H., Su, X.S., Ge, S.X., Zhang, S.Y. \& Xia, N.S. (2017). Epidemics and aetiology of hand, foot and mouth disease in Xiamen, China, from 2008 to 2015. Epidemiology and Infection 145: 1865-1874. https://doi.org/10.1017/S0950268817000309

Iwai, M., Masaki, A., Hasegawa, S., Obara, M., Horimoto, E., Nakamura, K., Tanaka, Y., Endo, K., Tanaka, K. \& Ueda, J. (2009). Genetic changes of coxsackievirus A16 and enterovirus 71 isolated from hand, foot, and mouth disease patients in Toyama, Japan between 1981 and 2007. Japanese Journal of Infectious Diseases 62: 254-259.

Mirand, A., Le Sage, F.V., Pereira, B., Cohen, R., Levy, C., Archimbaud, C., Peigue-Lafeuille, H., Bailly, J.L. \& Henquell, C. (2016). Ambulatory pediatric surveillance of hand, foot and mouth disease as signal of an outbreak of coxsackievirus A6 infections, France, 2014-2015. Emerging Infectious Diseases 22: 1884-1893. https://dx.doi.org/10.3201/ eid2211.160590

$\mathrm{MOH}, 2019$. Health Facts 2019. https://www.moh.gov.my/moh/ resources/Penerbitan / Penerbitan\%20Utama/ HEALTH\%20FACTS/Health\%20Facts\%202019_Booklet.pdf. Accessed 27 November 2020.

Nijhuis, M., van Maarseveen, N., Schuurman, R., Verkuijlen, S., de Vos, M., Hendriksen, K. \& van Loon, A.M. (2002). Rapid and sensitive routine detection of all members of the genus Enterovirus in different clinical specimens by real-time PCR. Journal Clinical Microbiology 40: 3666-3670. https://doi.org/10.1128/JCM.40.10.3666-3670.2002

Nix, W.A., Oberste, M.S. \& Pallansch, M.A. (2006). Sensitive, seminested PCR amplification of VP1 sequences for direct identification of all enterovirus serotypes from original clinical specimens. Journal of Clinical Microbiology 44: 26982704. https://doi.org/10.1128/JCM.00542-06

Puenpa, J., Mauleekoonphairoj, J., Linsuwanon, P., Suwannakarn, K., Chieochansin, T., Korkong, S., Theamboonlers, A. \& Poovorawan, Y. (2014). Prevalence and characterization of enterovirus infections among pediatric patients with hand foot mouth disease, herpangina and influenza like illness in Thailand, 2012. PLoS One 9: e98888. https://doi. org/10.1371/journal.pone.0098888

Robinson, C.C., Willis, M., Meagher, A., Gieseker, K.E., Rotbart, H. \& Glode, M.P. (2002). Impact of rapid polymerase chain reaction results on management of pediatric patients with enteroviral meningitis. Pediatric Infectious Disease Journal 21: 283-286.

Rose, T.M., Henikoff, J.G. \& Henikoff, S. (2003). CODEHOP (COnsensus-DEgenerate Hybrid Oligonucleotide Primer) PCR primer design. Nucleic Acids Research 31: 3763-3766. https://doi.org/10.1093/nar/gkg524

Solomon, T., Lewthwaite, P., Perera, D., Cardosa, M.J., McMinn, P. \& Ooi, M.H. (2010). Virology, epidemiology, pathogenesis, and control of enterovirus 71. Lancet Infectious Diseases 10: 778-790. https://doi.org/10.1016/S1473-3099(10)70194-8

Tan, C.W., Tee, H.K., Lee, M.H.P., Sam, I.C. \& Chan, Y.F. (2016). Enterovirus A71 DNA-launched infectious clone as a robust reverse genetic tool. PLoS One 11: e0162771. https://doi. org/10.1371/journal.pone.0162771
Thanh, T.T., Anh, N.T., Tham, N.T., Van, H.M., Sabanathan, S., Qui, P.T., Ngan, T.T., Van, T.T., Nguyet, L.A. \& Ny, N.T. (2015). Validation and utilization of an internally controlled multiplex Real-time RT-PCR assay for simultaneous detection of enteroviruses and enterovirus A71 associated with hand foot and mouth disease. Virology Journal 12: 85. https://doi.org/10.1186/s12985-015-0316-2

Upala, P., Apidechkul, T., Suttana, W., Kullawong, N., Tamornpark, R. \& Inta, C. (2018). Molecular epidemiology and clinical features of hand, foot and mouth disease in northern Thailand in 2016: a prospective cohort study. BMC Infectious Diseases 18: 630. https://doi.org/10.1186/ s12879-018-3560-4

Van Tu, P., Thao, N.T.T., Perera, D., Truong, K.H., Tien, N.T.K., Thuong, T.C., Ooi, M.W., Cardosa, M.J. \& McMinn, P.C. (2007). Epidemiologic and virologic investigation of hand, foot, and mouth disease, southern Vietnam, 2005. Emerging Infectious Diseases 13: 1733-1741. https://doi.org/10.3201/ eid1311.070632

Wei, S.H., Huang, Y.P., Liu, M.C., Tsou, T.P., Lin, H.C., Lin, T.L., Tsai, C.Y., Chao, Y.N., Chang, L.Y. \& Hsu, C.M. (2011). An outbreak of coxsackievirus $A 6$ hand, foot, and mouth disease associated with onychomadesis in Taiwan, 2010. BMC Infectious Diseases 11: 346. https://doi.org/10.1186/ 1471-2334-11-346

Weng, Y., Chen, W., He, W., Huang, M., Zhu, Y. \& Yan, Y. (2017). Serotyping and genetic characterization of hand, foot, and mouth disease (HFMD)-associated enteroviruses of non-EV71 and non-CVA16 circulating in Fujian, China, 20112015. Medical Science Monitor 23: 2508-2518. https://doi.org/ $10.12659 / \mathrm{msm} .901364$

Yang, Q., Gu, X., Zhang, Y., Wei, H., Li, Q., Fan, H., Xu, Y., Li, J., Tan, Z. \& Song, Y. (2018). Persistent circulation of genotype D coxsackievirus A2 in mainland of China since 2008. PLoS One 13: e0204359. https://doi.org/10.1371/journal.pone. 0204359 